\title{
Coding Bias in Respiratory Tract Infections May Obscure Inappropriate Antibiotic Use
}

\author{
Kathryn A. Martinez, PhD, MPH ${ }^{7}$, Mark Rood, $\mathrm{MD}^{2}$, and Michael B. Rothberg, $\mathrm{MD}, \mathrm{MPH}^{7}$ \\ ${ }^{1}$ Center for Value-Based Care Research, Cleveland Clinic, Cleveland, OH, USA; ${ }^{2}$ Department of Family Medicine, Cleveland Clinic, Cleveland, \\ $\mathrm{OH}, \mathrm{USA}$
}

J Gen Intern Med 34(6):806-8

DOI: $10.1007 / \mathrm{s} 11606-018-4823-\mathrm{x}$

(C) Society of General Internal Medicine 2019

\section{INTRODUCTION}

Inappropriate prescription of antibiotics for respiratory tract infections (RTIs) is common. ${ }^{1}$ To promote antibiotic stewardship, some quality improvement interventions aim to reduce inappropriate prescribing by monitoring individual physicians' prescribing rates for particular diagnoses. ${ }^{2}$

While over $90 \%$ of sinusitis is viral, ${ }^{3}$ antibiotics are sometimes indicated, whereas they are always inappropriate for viral upper respiratory infections, rhinitis or bronchitis. In order to avoid scrutiny when prescribing inappropriate antibiotics, physicians may diagnose a cold as sinusitis. Such coding bias has been hypothesized, ${ }^{4}$ but has not been specifically evaluated. Our objective was to assess differences in RTI coding for particular diagnoses by physician antibiotic prescribing rates.

\section{METHODS}

This study uses data from a large nationwide direct to consumer telemedicine platform ${ }^{5}$ and includes encounters with patients aged $\geq 18$ years completed between January 2013 and August 2016. Physicians recorded RTI diagnoses using ICD-9 and ICD-10 codes. Diagnoses were categorized as sinusitis, pharyngitis, bronchitis, and other RTI (including RTI not otherwise specified, influenza and rhinitis). Antibiotic receipt was determined by National Drug Codes recorded by the telemedicine physicians. This study was approved by Cleveland Clinic's Institutional Review Board.

We grouped physicians into quartiles of antibiotic prescribing for RTIs by generating an adjusted mean prescribing rate for each physician, using a mixed effects regression model. Patients are essentially assigned to physicians at random, so rates of specific illnesses should be evenly distributed among them. Nevertheless, we adjusted the model for patient sex, age, geographic region, and whether they provided insurance information. After assigning individual physicians to antibiotic prescribing quartiles, we assessed differences in (1) the proportion of specific RTI diagnoses and (2) antibiotic prescribing

Published online January 16, 2019 for specific RTI diagnoses, by prescribing quartiles. Analyses were conducted in Stata 14.

\section{RESULTS}

The sample included 13,025 RTI encounters with 105 physicians. Mean patient age was 39 years (interquartile range $31-$ 47), $60 \%$ were female, and $73 \%$ provided insurance information. Nearly half (49\%) of encounters were coded as sinusitis, $13 \%$ as pharyngitis, $12 \%$ as bronchitis, and $25 \%$ as other RTI. The overall mean adjusted antibiotic prescribing rate was $40 \%$ for the lowest prescribing quartile (quartile 1) and $87 \%$ for the highest prescribing quartile (quartile 4).

Physicians' overall antibiotic prescribing rate was associated with the frequency of diagnosing a specific RTI (Fig. 1). Physicians in quartile 1 diagnosed $42 \%$ of patients with other RTIs and $35 \%$ of patients with sinusitis, compared to physicians in quartile 4 , who diagnosed $59 \%$ of patients with sinusitis and $9 \%$ with other RTIs $(p<0.001)$. Overall antibiotic prescribing quartile was associated with antibiotic prescribing for specific diagnoses as well, with physicians in quartile 4 prescribing at the highest rate for all RTI types (Fig. 2).

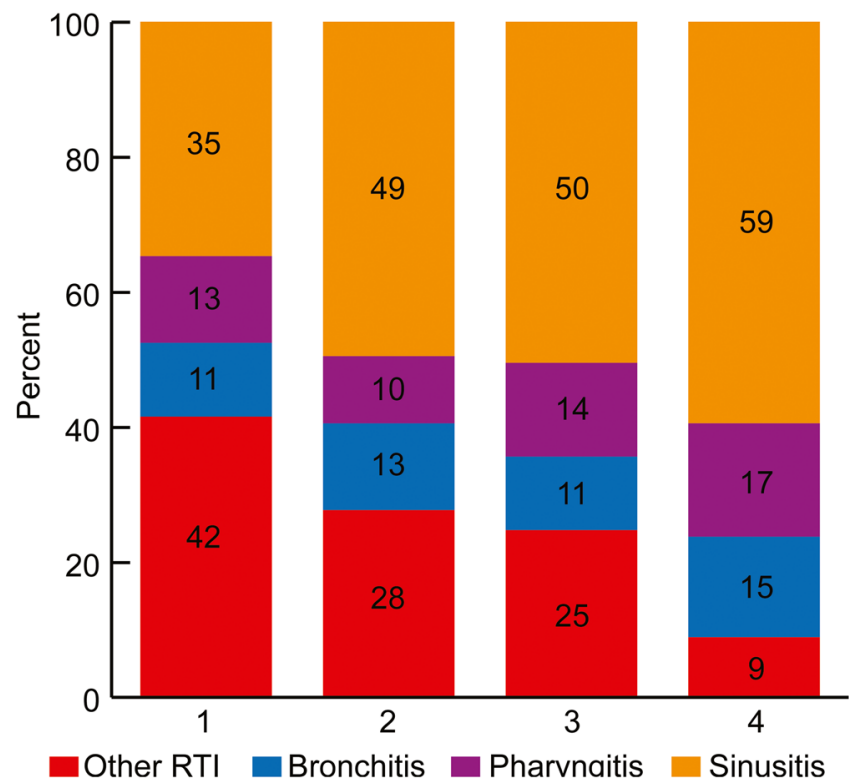

Figure 1 Distribution of RTI diagnoses by physician antibiotic quartiles. 

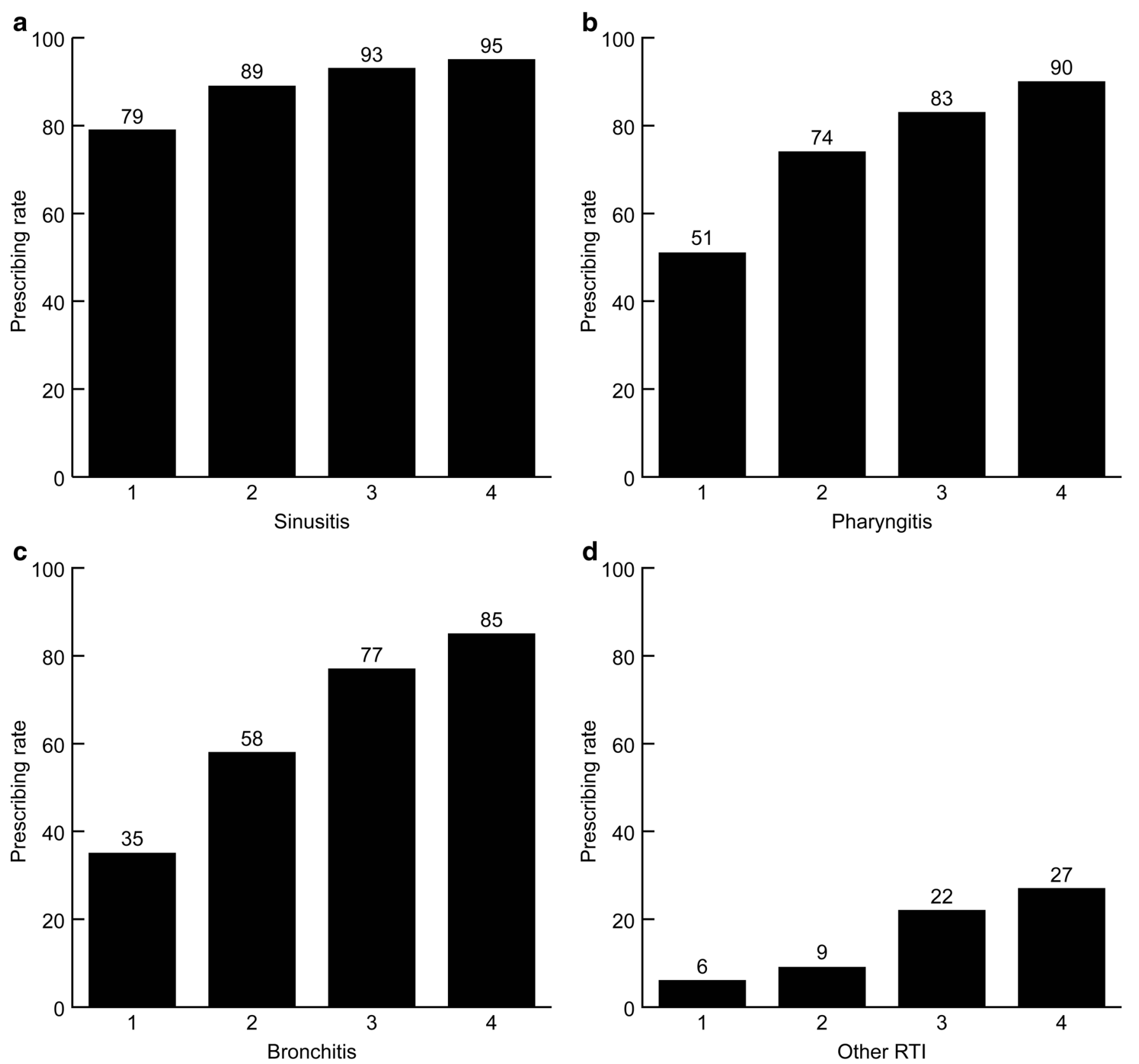

Figure 2 Antibiotic prescribing rate for each diagnosis by physician overall antibiotic prescribing quartile.

\section{DISCUSSION}

High prescribing physicians in our sample were the most likely to code RTIs as sinusitis while low prescribing physicians were the least likely to code sinusitis, suggesting evidence of coding bias. The extent to which this coding bias is a conscious behavior is unknown. Physicians who prescribe antibiotics at a high rate may simply be more inclined to diagnose patients with antibiotic-indicated conditions. However, we also found these physicians prescribed antibiotics at a higher rate for all RTI types.

That higher prescribers are also more likely to diagnose sinusitis was recently documented in an outpatient setting. ${ }^{6}$ While the distinction between appropriate versus inappropriate prescribing is important, studies examining variation in antibiotic use for RTIs should include all diagnoses. Excluding sinusitis (and pharyngitis) because antibiotics are sometimes warranted may inadvertently exclude most inappropriate prescribing.

Our study had some limitations. These include being conducted in the telemedicine setting, which may differ from traditional outpatient care, and our inability to account for antibiotic stewardship efforts, which may have taken place during the study period.

Physicians who prescribe a lot of antibiotics also diagnose sinusitis at a disproportionately high rate. Excluding sinusitis from studies of antibiotic overuse will therefore overlook a large share of inappropriate prescribing. Benchmarking physicians on their RTI coding in addition to antibiotic prescribing may help to mitigate coding bias. 
Corresponding Author: Kathryn A. Martinez, PhD, MPH; Center for Value-Based Care Research, Cleveland Clinic, Cleveland, OH, USA (e-mail: martink12@ccf.org).

\section{Compliance with Ethical Standards:}

This study was approved by Cleveland Clinic's Institutional Review Board.

Conflict of Interest: The authors declare that they do not have a conflict of interest.

Publisher's note: Springer Nature remains neutral with regard to jurisdictional claims in published maps and institutional affiliations.

\section{REFERENCES}

1. Spellberg B, Guidos R, Gilbert $\mathbf{D}$, et al. The epidemic of antibioticresistant infections: a call to action for the medical community from the
Infectious Diseases Society of America. Clin Infect Dis. 2008;46(2):155164. https://doi.org/10.1086/524891.

2. Tonkin-Crine SK, Tan PS, van Hecke $\mathbf{O}$, et al. Clinician-targeted interventions to influence antibiotic prescribing behaviour for acute respiratory infections in primary care: an overview of systematic reviews. Cochrane Database Syst Rev. 2017;9:CD012252. https://doi.org/10. 1002/14651858.CD012252.pub2.

3. Chow AW, Benninger MS, Brook I, et al. Executive Summary: IDSA Clinical Practice Guideline for Acute Bacterial Rhinosinusitis in Children and Adults. Clin Infect Dis. 2012;54(8):1041-1045. https://doi.org/10. 1093/cid/cir1043.

4. Linder JA, Singer DE. Desire for antibiotics and antibiotic prescribing for adults with upper respiratory tract infections. J Gen Intern Med. 2003; 18(10):795-801.

5. Martinez KA, Rood M, Jhangiani N, et al. Patterns of Use and Correlates of Patient Satisfaction with a Large Nationwide Direct to Consumer Telemedicine Service. J Gen Intern Med. 2018. https://doi.org/10.1007/ s11606-018-4621-5.

6. Manne M, Deshpande A, Hu B, et al. Provider Variation in Antibiotic Prescribing and Outcomes of Respiratory Tract Infections. South Med J. 2018;111(4):235-242. https://doi.org/10.14423/SMJ. 0000000000000795 . 